PROCEEDINGS OF THE

AMERICAN MATHEMATICAL SOCIETY

Volume 124, Number 10, October 1996

\title{
A COUNTEREXAMPLE TO CARTAN'S CONJECTURE ON HOLOMORPHIC CURVES OMITTING HYPERPLANES
}

\author{
ALEXANDRE EREMENKO
}

(Communicated by Albert Baernstein II)

\begin{abstract}
In his 1928 thesis H. Cartan proved a theorem which can be considered as an extension of Montel's normality criterion to holomorphic curves in complex projective plane $\mathbf{P}^{2}$. He also conjectured that a similar result is true for holomorphic curves in $\mathbf{P}^{n}$ for any $n$. A counterexample to this conjecture is constructed for any $n \geq 3$.
\end{abstract}

The following theorem of Borel may be considered as an extension of Picard's theorem to holomorphic mappings of the complex plane $\mathbf{C}$ to complex projective space.

Borel's Theorem. Let $f_{1}, \ldots, f_{p}$ be a system of entire functions without zeros and

$$
f_{1}+\ldots+f_{p}=0 .
$$

Then the set of indices $\{1, \ldots, p\}$ can be partitioned into disjoint subsets $\{I\}$ such that $|I| \geq 2$, and for every $I$ the functions $f_{j}, j \in I$, are proportional and their sum is zero.

According to the so-called Bloch principle, to every theorem of Picard type should correspond a Montel-type theorem for families of functions in the unit disk. The following statement is known as

Cartan's Conjecture $([2,3])$. Let $\mathcal{F}$ be an infinite family of $p$-tuples of holomorphic functions $f=\left(f_{1}, \ldots, f_{p}\right)$ without zeros in the unit disk $\mathbf{U}$ satisfying the Borel equation (1).

Then there exists an infinite subsequence $\mathcal{L}$ having the following property.

There exists a partition of indices $P=\{1, \ldots, p\}$ into disjoint sets $\{S\}$ and each $S$ contains a subset $I$ with at least two elements, which may be equal to $S$ itself. These satisfy the following properties for $f \in \mathcal{L}$ :

(i) For each $S$ and $j, k \in I \subset S$ the sequence $\left\{f_{j} / f_{k}\right\}$ is convergent (uniformly on compacta, to a non-zero function).

(ii) If $j \in S \backslash I$ and $k \in I \subset S$ then $f_{j} / f_{k}$ converges to 0 .

(iii) Given $k \in I \subset S$,

$$
\sum_{j \in I} f_{j} / f_{k} \text { converges to } 0 .
$$

Received by the editors March 29, 1995.

1991 Mathematics Subject Classification. Primary 30D45; Secondary 32H30.

(C)1996 American Mathematical Society 
When $p=3$ the statement is (almost) equivalent to the Montel theorem, which asserts that a family of meromorphic functions in the unit disk omitting three given values is normal. Cartan [2], see also [3, Ch. VIII], proved a partial result:

Let $\mathcal{F}$ be as above. Then there exists a subsequence $\mathcal{L} \subset \mathcal{F}$ having one of the following properties:

(a) The full set $P$ of indices satisfies (i), (ii) and (iii) (with single set $S=P$ ), or

(b) There are two disjoint subsets $S_{1}$ and $S_{2}$ in $P$, each containing at least two elements, satisfying the three conditions (i), (ii) and (iii).

The point is that $S_{1}$ and $S_{2}$ in (b) may not cover the whole set of indices $P$. This result implies that Cartan's conjecture is true for $p=3$ and $p=4$ [2]. We show that it fails for $p=5$.

Example. It is convenient to work in the rectangle $R=\{x+i y:|x|<\pi, 0<$ $y<1\}$ instead of the unit disk. For every natural integer $n>12>4 e$ consider the function $h(z)=h_{n}(z)=\exp (n \exp i z), z \in R$. We have

$$
\log \left|h_{n}(x+i y)\right|=n \cos x \exp (-y) .
$$

The set $\left\{z \in R:\left|h_{n}(z)\right|<3\right\}$ consists of two components: left and right. We denote the right component by $D_{n}$ so that as $n \rightarrow \infty, D_{n} \rightarrow R \cap\{x \geq \pi / 2\}$. Choose a diffeomorphism $p$ of the disk $\{w:|w| \leq 3\}$ onto itself with the following properties:

$$
\begin{gathered}
p(w)=w, \quad|w|=3, \\
p(0)=1
\end{gathered}
$$

and

$$
p \text { is conformal for }|w|<2 \text {. }
$$

Put

$$
\tilde{G}_{n}(z)= \begin{cases}p \circ h_{n}(z), & z \in D_{n}, \\ h_{n}(z), & z \in R \backslash D_{n} .\end{cases}
$$

Then we can find a diffeomorphism $\phi_{n}: R \rightarrow R$, continuous in $\bar{R}$ with

$$
\phi_{n}(0)=0, \quad \phi_{n}( \pm \pi)= \pm \pi
$$

such that

$$
G_{n}=\tilde{G}_{n} \circ \phi_{n}^{-1}
$$

is holomorphic in $R$. This $\phi_{n}$ is obtained by solving a Beltrami equation [1]

$$
\frac{\partial \phi_{n}}{\partial \bar{z}}=\mu \frac{\partial \phi_{n}}{\partial z},
$$

where $\mu$ is a smooth function, $|\mu(z)| \leq c \leq 1, z \in R, c$ an absolute constant, and

$$
\operatorname{supp} \mu=K_{n}=\left\{z \in R: \Re z>0,2 \leq\left|h_{n}(z)\right| \leq 3\right\} .
$$

We claim that

$$
\phi_{n}(z)-z \rightarrow 0, \quad n \rightarrow \infty
$$

uniformly on $R$. Indeed, $\left\{\phi_{n}\right\}$ is a family of quasiconformal homeomorphisms of $R$ with uniformly bounded dilatation, so this family is precompact (the topology of 
uniform convergence). Any limit function $\phi$ of the family is conformal everywhere in $R$ except perhaps the segment

$$
K=\{\pi / 2+i t: 0<t<1\}=\lim _{n \rightarrow \infty} K_{n} .
$$

But $K$ is a removable singularity for homeomorphisms conformal in the complement of $K$. So $\phi$ is a conformal automorphism of $R$ and (2) implies that $\phi=$ id. This proves (4). Notice that $G_{n}-1$ has no zeros in $R \cap\{x>0\}$ and $G_{n}$ has no zeros in $R \cap\{x<0\}$. It follows from (4) that

$$
\log \left|G_{n}(x+i y)-1\right|=(n+o(1)) \cos x \exp (-y), \quad x>0
$$

and

$$
\log \left|G_{n}(x+i y)\right|=(n+o(1)) \cos x \exp (-y), \quad x<0,
$$

when $n \rightarrow \infty$ uniformly on $R$. Now we define $H_{n}$ by

$$
G_{n}+H_{n}=1 .
$$

Asymptotic equalities (5) and (6) imply respectively

$$
\log \left|H_{n}(x+i y)\right|=(n+o(1)) \cos x \exp (-y), \quad x>0
$$

and

$$
\log \left|H_{n}(x+i y)-1\right|=(n+o(1)) \cos x \exp (-y), \quad x<0,
$$

as $n \rightarrow \infty$ uniformly on $R$.

Now we set $a=\pi-1 /(e+1)$ and define

$$
\begin{gathered}
f_{n}^{1}(z)=\exp \{n(z+a)\}, f_{n}^{2}(z)=\exp \{n(-z+a)\}, \\
f_{n}^{3}=G_{n}-f_{n}^{1}, f_{n}^{4}=H_{n}-f_{n}^{2}, f_{n}^{5}(z) \equiv-1 .
\end{gathered}
$$

From this definition and (7) follows that (1) is satisfied. Furthermore we have in view of (5), (6), (8) and (9)

$$
\left|G_{n}\right|<\left|f_{n}^{1}\right| \quad \text { and } \quad\left|H_{n}\right|<\left|f_{n}^{2}\right| \quad \text { in } \quad R
$$

for $n$ large enough.

Inequalities (10) show that all five functions $f^{j}$ are zero-free in $R$ if $n$ is large enough.

Now we show that the conclusion of Cartan's conjecture is not valid for the functions of our sequence. This is because $f_{n}^{5}$ cannot be in the same class $S$ with any other function $f_{n}^{j}, 1 \leq j \leq 4$. Indeed, when $j$ is odd we have

$$
\log \left|f_{n}^{j}(z)\right|=(n+o(1))(\Re z+a), \quad n \rightarrow \infty,
$$

so

$$
f_{n}^{j}\left(-\pi+\frac{1}{2(e+1)}+\frac{i}{2}\right) \rightarrow 0 \quad \text { and } \quad f_{n}^{j}(i / 2) \rightarrow \infty, \quad n \rightarrow \infty .
$$

A similar argument works for even $j$. In this case

$$
f_{n}^{j}\left(\pi-\frac{1}{2(e+1)}+\frac{i}{2}\right) \rightarrow 0 \quad \text { and } \quad f_{n}^{j}(i / 2) \rightarrow \infty, \quad n \rightarrow \infty .
$$

So $f_{n}^{5} \equiv-1$ cannot be included in any class $S$ described in (i) and (ii) of Cartan's conjecture. 
Remarks. The simplest counterexample for any $p>6$ can be constructed by adding non-zero constant functions $f_{n}^{j}$ with the properties

$$
\sum_{j=6}^{p} f_{n}^{j}=0
$$

and $\left|f_{n}^{j}\right|=b^{-n}, 6 \leq j \leq p$, where $1<b<\exp \{1 /(e+1)\}$. These new functions may be included in one class $S$ with $f_{n}^{5}$ but then (iii) fails for this class. Our example for $p=5$ shows that even a partition into classes $S$, card $S \geq 2$, which satisfy (i) and (ii), is impossible. Examples with this property can also be constructed for any $p>5$.

The author thanks David Drasin, who made many helpful suggestions, and V. Lin for illuminating discussions.

\section{REFERENCES}

1. Lars V. Ahlfors, Lectures on Quasiconformal Mappings, D. Van Nostrand, Princeton, NJ 1966. MR 34:336

2. Henri Cartan, Sur les systèmes de fonctions holomorphes à variétés linéaires lacunaires et leurs applications, Ann. École Normale Supèr., 45 (1928), 255-346.

3. Serge Lang, Introduction to Complex Hyperbolic Spaces, Springer-Verlag, NY, 1987. MR 88f:32065

Department of Mathematics, Purdue University, West Lafayette, Indiana 47907

E-mail address: eremenko@math.purdue.edu 